\title{
Simulation Analysis of Control Strategy and Commutation Failure in HVDC Transmission System
}

\author{
Peizhe $\mathrm{Li}^{1,}$, , Liangeng $\mathrm{Ban}^{1}$ and Huawei Wang ${ }^{1}$ \\ ${ }^{1}$ China Electric Power Research Institute, Beijing 100192, China; \\ azjklpz@163.com
}

\begin{abstract}
Keywords: electromagnetic transient simulation, HVDC transmission system model, commutation failure, operation condition
\end{abstract}

\begin{abstract}
By means of electromagnetic transient simulation program, a detailed model of High Voltage Direct Current (HVDC) power transmission system actually operated in current power grid is built, and detailed variation of electric quantities, the duration of commutation failure and its impacts on AC power transmission grid as well as DC block during the commutation failure process in that system are analyzed. Research results show that commutation failure leads to severe fluctuation of such electrical quantities as DC current, DC voltage and DC power, however the duration of the fluctuations usually is no longer than $100 \mathrm{~ms}$, so its impact on AC power system is transitory, meanwhile there is low probability of DC block caused by commutation failure.
\end{abstract}

\section{Introduction}

HVDC power transmission has the advantages of large capacity and low loss, quick and flexible regulation or reverse of transmission power, high reliability, and strong asynchronous communication capability. Therefore, it is widely used in large and remote power grid interconnection as well as large capacity power transmission ${ }^{[1]}$. As the most common failure in HVDC transmission system, commutation failure leads to the drop of DC voltage and the sudden increase of current, which influence the operation of system, even cause the blocking or pole blocking of valve group ${ }^{[2]}$.

References [3,4] analyze the main influencing factor of inverter commutation failure in HVDC system in detail. Based on sensitivity analysis, the general principle of commutation failure is concluded, and the basic control and prevention measures are provided. Reference [5] also studies the mechanism, factors and judgment of commutation failure, and points out the main problems. However, its analysis of commutation failure in single DC feed-in system is not comprehensive.

In this paper, the detailed model of practical HVDC transmission system is built through electromagnetic transient simulation program. Based on the model, the DC voltage, current, active power and exchanged of inverter side in commutation failure process are analyzed in detail, as well as the duration of failure under different fault conditions and the its influence on power grid.

\section{Definition of Commutation Failure of DC Inverter}

In the inverter, during the action time of reverse voltage, if the commutation process of the valve has not been completed, or it fails to restore the blocking ability, when the voltage becomes positive, the commutation valve will be conducted again, called as commutation failure.

Causes of commutation failure fall into two categories: the internal fault in HVDC system, and the influence of fault or disturbance in AC system of receiving end. For multiple DC feed-in system, DC transmission system failure will only cause the commutation failure in DC system, but the fault or disturbance of AC system may affect all the nearby DC system, causing commutation failures in all the DC transmission system at the same time.

For short-term commutation failure, after the fault is cleared, the DC inverter usually can return to normal commutation. For long-term continuous failure, it may cause the blocking of DC protection system, thus leading to a great impact on transmission system. 


\section{Simulation of System Operation and Commutation Failure}

\subsection{AC System.}

Modelling of AC System in electromagnetic transient program includes keep generating units, equivalent units, transmission lines and load considering static characteristics, as follows. Generator and its regulator model: the generating units and equivalent units in system are described by generator models in PSCAD/EMTDC software. Generator parameters are input according to the electromechanical transient program BPA. Transmission line model: usually the distributed parameter line model is used to get the frequency response and distribution characteristics of transmission lines, and realize the purpose of dividing subsystems during large-scale simulation. Load model: in power system analysis, quadratic polynomials are commonly used to approximately express the voltage characteristics of load. The zero, monomial and quadratic terms are equal to constant power load, constant current load, and constant impedance load separately.

\subsection{Parameters of DC Circuit.}

The rated current of DC system is 4750A. Considering the input of reserve cooling equipment, the maximum current of DC system would reach 5000A in the highest environmental temperature. In bipolar operating mode, the rated transmission power of $1100 \mathrm{kV}$ system is $10450 \mathrm{MW}$.

\subsection{Simulation of Commutation Failure.}

For DC transmission system, the failure of AC system in receiving end would cause the voltage drop of converter bus in inverter station. If the drop is too large, the commutation failure of inverter in DC system would often occur. During the commutation failure, the sharp rise of DC current steep drop of DC voltage would cause large impact to AC/DC power transmission system. Due to the short duration of failure (generally no more than $100 \mathrm{~ms}$ ), with the fault clearance, the transmission system usually can switch back to normal and keep stable. Single-phase and three-phase fault cases in AC system of receiving end are concerned in this section.

Single-phase instantaneous and three-phase permanent metallic ground fault in AC line of receiving end are simulated. After $100 \mathrm{~ms}$, the fault phases jump. In $\pm 1100 \mathrm{kV}$ HVDC system, constant power control of rectifier side is used, while predictive turn-off angle control of inverter side is used during the calculation. The DC transmission power is $10450 \mathrm{MW}$. The short-circuit currents of sending end and receiving end are set to 63kA. The effective short-circuit ratios of sending and receiving system are 7.4 and 4.9 separately.

When phase A ground fault happens in inverter station of receiving end, forecast of commutation failure will be quickly launched, reducing the trigger angle to increase the commutation margin. During the fault, the DC voltage and power of inverter station fall sharply (lasts $10 \mathrm{~ms}$ ). The maximum current reaches $11800 \mathrm{~A}$, and the trigger angle decreases to $111^{\circ}$. In a short time, the inverter station absorbs reactive power from AC system, reaching 5300Mvar at most. After the fault is cleared, due to the slower recovery speed of DC voltage than the AC voltage, the DC system injects massive reactive power into AC system, causing the short-term voltage rise of converter bus.

In the rectifier station, the DC voltage drops to $-1115 \mathrm{kV}$, the current rises to $10700 \mathrm{~A}$ at most, and the trigger angle moves to $134.8^{\circ}$ at most. The rectifier station absorbs much reactive power from AC system, reaching 8212Mvar at most. It leads to the short-term voltage drop of converter bus drop, with the minimum peak value of phase voltage reaching $475 \mathrm{kV}(0.78$ p.u., and the basic value of phase voltage is $612.3 \mathrm{kV}$ ) for $40 \mathrm{~ms}$. Under the action of current regulator, the DC current drops rapidly to 0 at least. And the reactive power consumed in converter falls sharply. With the function of all the AC filters, massive reactive power is offered to AC system, causing the short-term voltage rise of converter bus. The results can be seen in Fig.1. 

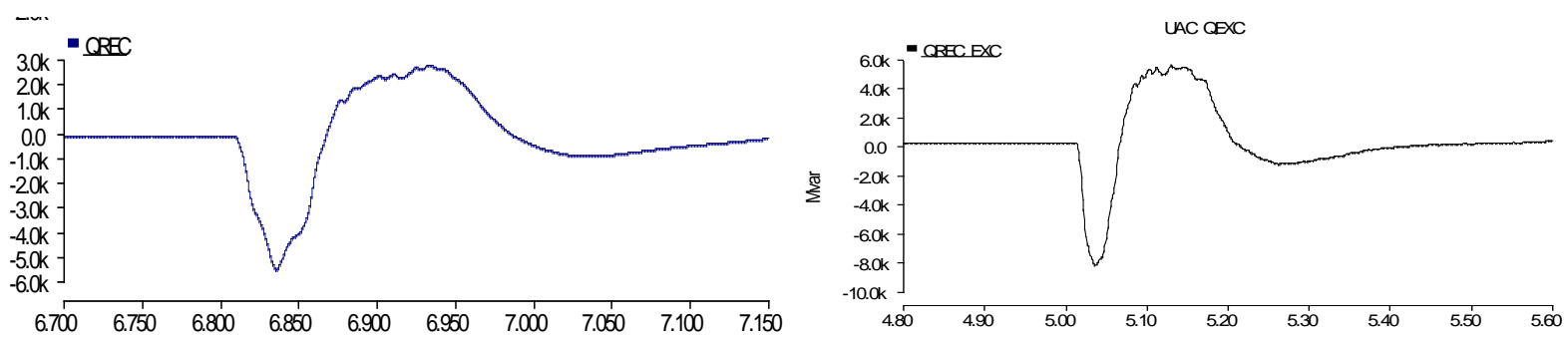

(a) Rectifier side
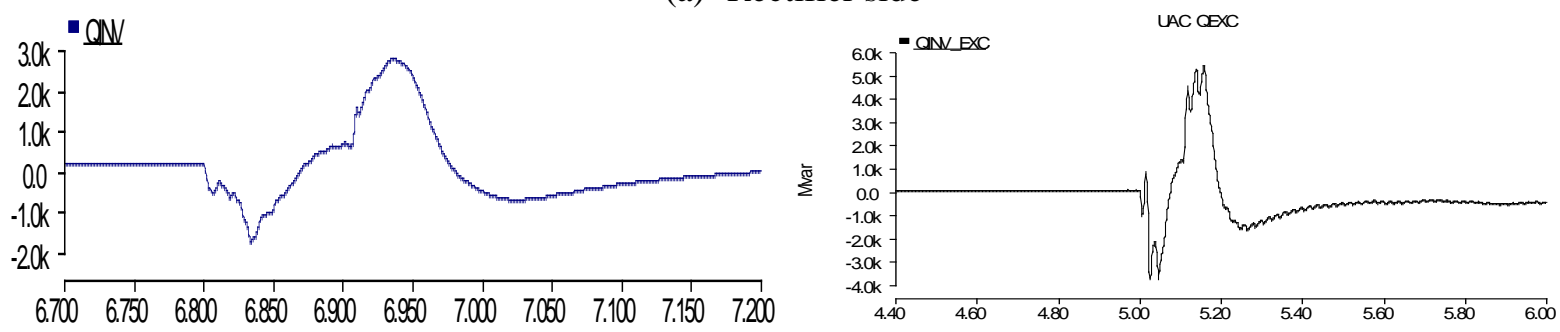

(b) Inverter side

Fig. 1 The variation of reactive power during commutation failure and recovery period of $800 \mathrm{kV}$ and $+1100 \mathrm{kV}$ HVDC system

In addition, the effects of AC system of receiving end under different short-circuit ratios are studied. There are two cases that the short-circuit currents of sending end are 40kA and 16.7kA (short-circuit ratio 4.5 and 4.5), while the short-circuit currents of receiving end are 40kA and 24.5 kA separately (short-circuit ratio 3.5 and 3.5).The results can be seen in Fig.2.


Fig. 2 Simulation wave of the AC system under different short-circuit ratio (3.5 and 2.1)

During the commutation failure, the reactive power absorbed by rectifier and inverter station is associated with DC current and trigger angle. When the effective short-circuit ratio is 7.4 , the maximum current reaches $10700 \mathrm{~A}$, and the maximum trigger angle reaches $134.8^{\circ}$. The absorbed reactive power reaches $8200 \mathrm{Mvar}$ at most. When the effective short-circuit ratio decreases to 1.6, the maximum current reaches $8813 \mathrm{~A}$, and the maximum trigger angle reaches $114.1^{\circ}$. The absorbed reactive power decreases significantly.

In the inverter station, the absorbed reactive power keeps roughly constant under different short -circuit ratio. During the recovery of commutation failure, due to the slower speed of DC current than the AC voltage, the output reactive power from AC filters rapidly increases, while the DC current gradually recovers with the increase of DC voltage. In a short time, the converter station injects massive reactive power into AC system, and the level of reactive power is related to DC current and trigger angle during the commutation failure. 


\section{Summary}

1) During the commutation failure, the absorbed reactive power is associated with the magnitude of DC current and trigger angle. During the recovery, the injected reactive power to AC system is associated with the magnitude of DC current.

2) When the effective short-circuit ratio falls to 2.1, under single-phase fault in inverter station, the absorbed reactive power during the failure can lead to significant voltage drop of non-fault phase in converter bus. And during the recovery process, the voltage of converter bus increases significantly.

3) For single-phase fault happened in weak AC system of receiving end, after the fault is cleared, the voltage distortion is serious. Furthermore, the commutation failure forecast starts for a long time (600ms), causing the increase of turn-off angle in inverter station. Therefore, it will absorb a large amount of reactive power from the system, extending the recovery time of DC system.

4) According to the calculation and simulation, due to the large leakage resistance of converter transformer in $\pm 1100 \mathrm{kV}$ DC transmission system, commutation failure and even bipolar commutation failure easily occurs during the recovery process of fault in DC line. Thus it is suggested to appropriately increase the rated turn-off angle of inverter station. Furthermore, when the single twelve pulse converter shuts down and exits urgently due to the fault, commutation failure is also prone to occur because of the current increase caused by short-term overload.

\section{References}

[1] Wu Ping, Lin Weifang, Sun Huadong, et al. Research and electromechanical transient simulation on mechanism of commutation failure in multi-infeed HVDC power transmission system[J]. Power System Technology. Vol. 36 (2012) No.5, p. 269-274 (in Chinese).

[2] Li Xinnian, Li Tao, Wang Jingfang, et al. Impacts of $800 \mathrm{kV}$ DC power transmission from Yunnan to Guangdong on stability of China Southern Power Grid[J]. Power System Technology. Vol. 33 (2009), No.20, p. 21-26 (in Chinese).

[3] Li Xinnian, Wang Mingxin.Issues and measures of commutation failure in inverter station[R]. Beijing: China Electric Power Research Institute, 2011.

[4] Wang Jingfang, Yu Jun, Li Xinnian, et al. Simulation and research of connected system in $800 \mathrm{kV}$ DC sending project[R]. Beijing: China Electric Power Research Institute, 2005.

[5] Wang Jingfang, Yu Jun, Li Xinnian, et al. PSCAD Calculation and research of multiple landing points DC sytem in China Southern Power Grid in 2010[R]. Beijing: China Electric Power Research Institute, 2006.

[6] Chen Shuyong, Li Xinnian, Yu Jun, et al. A method based on the sin-cos components detection mitigates commutation failure in HVDC[J]. Proceedings of the CSEE. Vol. 25 (2005), No.14, p.1-6 (in Chinese).

[7] Lu Pengfei, LiXinnian, Chen Lingfang, et al. A new method of preventing commutation failure in HVDC based on sin-coscomponents detection[C].International Conference on Electrical Engineering. YongPyong Korea: ICEE, 2006: 2-11. 